\title{
Relación de vegetación ribereña y propiedades del suelo en un afluente del río Tacotalpa, Tabasco, México
}

\section{Relationship between riparian vegetation and soil properties in a tributary of the river Tacotalpa, Tabasco, Mexico}

\author{
Victorio Moreno-Jiménez*, Ofelia Castillo-Acosta', Lilly Gama-Campillo², Joel Zavala-Cruz³ y Mario Arturo Ortiz-Pérez ${ }^{\dagger 4}$ \\ 1 Universidad Juárez Autónoma de Tabasco. División \\ Académica de Ciencias Biológicas. Laboratorio de \\ Ecofisiología Vegetal y Comunidades Vegetales. Vi- \\ llahermosa, Tabasco, México. \\ 2 Universidad Juárez Autónoma de Tabasco. División \\ Académica de Ciencias Biológicas. Laboratorio de \\ Ecología del Paisaje y Cambio Global. Villahermosa, \\ Tabasco, México. \\ 3 Colegio de Postgraduados, Campus Tabasco. Cár- \\ denas, Tabasco, México. \\ 4 Universidad Nacional Autónoma de México. Institu- \\ to de Geografía. Ciudad de México, México.
}

* Autor de correspondencia. victorm992@ahotmail.com

\section{RESUMEN}

La vegetación ribereña ha sido fuertemente fragmentada por acciones antrópicas modificando su estructura y sus funciones ecosistémicas. Se analizaron las variables de vegetación ribereña y su relación con las propiedades del suelo en un afluente del río Tacotalpa, Tabasco. A lo largo del cauce se seleccionaron tres zonas: baja, media y alta; en cada zona se establecieron ocho unidades de muestreo de $5 \mathrm{~m} \times 50 \mathrm{~m}$ y se censaron todos los árboles y arbustos con DAP $\geq 1 \mathrm{~cm}$. Se identificaron las especies y se generó información sobre el índice de valor de importancia (IVI), diversidad de especies (H') e índice de similitud (IS). En cada unidad de muestreo se colectó una muestra de suelo a una profundidad de $0 \mathrm{~cm}-30 \mathrm{~cm}$ y se analizaron las propiedades textura, $\mathrm{pH}$, materia orgánica $(\mathrm{MO})$, nitrógeno total $(\mathrm{Nt})$, fósforo asimilable $(\mathrm{P})$ y potasio $(\mathrm{K})$. Las variables se analizaron con los paquetes estadísticos SPSS y PAST. En las tres zonas de vegetación ribereña se registraron 1681 individuos, 38 familias y 131 especies; las familias Fabaceae, Euphorbiaceae y Moraceae fueron las más representativas. La diversidad fue similar en las tres zonas y la similitud osciló entre $31.3 \%$ y $53.3 \%$. Los contenidos de $\mathrm{Nt}$, MO y P fueron mayores en suelos de la zona alta con vegetación más conservada y menores en las zonas baja y media con vegetación secundaria; estas variables edáficas se correlacionaron positivamente con la altura de los árboles $(\mathrm{p}<0.01)$, siendo evidente el efecto benéfico de la interacción planta-suelo.

Palabras Clave: abundancia, composición, correlación, diversidad, estructura, nutrientes.

\section{ABSTRACT}

The riparian vegetation has been strongly fragmented by human actions modifying its structure and ecosystem functions. We analyzed the relationship between soil properties and riparian vegetation variables from a tributary of the Tacotalpa River in Tabasco. Three zones were selected along the course: low, medium and high; eight $5 \mathrm{~m} \times 50 \mathrm{~m}$ units were established on each sampling zone and all trees and shrubs with DAP $\geq 1 \mathrm{~cm}$ were counted. The species were identified and information about the importance value index (IVI), species diversity (H') and similarity index (IS) was generated. A soil sample was collected on each sampling unit on a depth of $0 \mathrm{~cm}-30 \mathrm{~cm}$, and texture properties, $\mathrm{pH}$, organic matter $(\mathrm{OM})$, total nitrogen $(\mathrm{Nt})$, available phosphorus $(\mathrm{P})$ and potassium $(\mathrm{K})$ were analyzed. All variables were analyzed using SPSS and PAST statistical packages. On the three riparian vegetation areas, 1681 individuals, 38 families and 131 species were recorded; the Fabaceae, Euphorbiaceae and Moraceae families were the most representative. The diversity was similar in all three zones and the similarity ranged between $31.3 \%$ and $53.3 \%$. The contents of $\mathrm{Nt}$, MO and P were higher in soil of the upper area with most preserved vegetation and lower in low and middle areas with secondary vegetation. These edaphic variables were positively correlated with tree height $(\mathrm{p}<0.01)$, being evident the beneficial effect of plant-soil interactions.

KEYWORDS: abundance, composition, correlation, diversity, structure, nutrients. 


\section{INTRODUCCIÓN}

La vegetación ribereña es uno de los ecosistemas más diversos y dinámicos (Guevara et al., 2008), forma un ecotono entre ecosistemas terrestres y acuáticos (Granados-Sánchez, Hernández-García y López-Ríos, 2006) y desempeña diversas funciones como: estabilidad en las márgenes de los ríos, retención de sedimentos, creación de hábitats para organismos acuáticos y terrestres (Camacho, Trejo y Bonfil, 2006). También ha sido uno de los ecosistemas más afectados por acciones humanas (Sunil, Somashekar y Nagaraja, 2010), generando cambios en la estructura y composición florística, favoreciendo la pérdida de diversidad biológica (Ede y Hunt, 2009). Actualmente se desconoce el porcentaje de pérdida de los bosques ribereños, pero se estima una disminución de $60 \%$ de las selvas húmedas tropicales del planeta (Llaven, 2013). En Tabasco, los bosques ribereños representan $1.37 \%$ de la superficie total de humedales y ocupan solamente $0.74 \%$ en la Sierra del estado (Barba-Macías, Rangel-Mendoza y Ramos-Reyes, 2006). Este ecosistema se caracteriza por desarrollarse en las orillas de ríos, arroyos y lagos (Díaz, Rueda, Acosta, Martínez y Castellanos, 2010; Sunil et al., 2010), sus propiedades estructurales y funcionales varían a través del gradiente altitudinal (Acosta, Mondragón y Alvarado, 2008). Crece sobre suelos Fluvisoles con buena fertilidad y bien drenados (Zavala-Cruz, Palma-López, Fernández, López y Shirma, 2011). Los suelos están condicionados por el tipo de roca, relieve, clima y vegetación (Sarr y Hibbs, 2007), lo que contribuye a variaciones en las comunidades vegetales (Decocq, 2002). El análisis de los cambios en la microtopografía y las variables edáficas textura y $\mathrm{pH}$, sugiere una alta correlación con la distribución de las especies vegetales y con las características de la estructura de las comunidades tropicales (CortésCastelán e Islebe, 2005). De la misma manera, se ha comprobado que el clima, la altitud, los factores topográficos y edáficos afectan el desarrollo del suelo, determinando la distribución vegetal a escala local (García y Vollmann, 2012).

\section{OBJETIVOS}

La presente investigación tiene por objetivo analizar las variables de vegetación ribereña y su relación con propiedades del suelo en tres zonas de un afluente del río Tacotalpa, para generar conocimiento que contribuya a la conservación y recuperación del bosque ribereño en el Parque Estatal de La Sierra en Tabasco. Para ello, se plantearon las preguntas siguientes: ¿cuál es la estructura y composición de especies vegetales en tres zonas del afluente en el Río Tacotalpa?, ¿existe alguna diferencia en la diversidad y propiedades del suelo en las tres zonas de estudio? y ¿qué propiedades del suelo influyen en las variables de vegetación ribereña en el área de estudio?

\section{MATERIALES Y MÉTODOS}

\section{Área de estudio}

Se ubica en un afluente del río Tacotalpa, en la Estación Biológica La Florida y el ejido Xicoténcatl, en el Parque Estatal La Sierra, municipio de Tacotalpa, Tabasco (Fig. 1). El afluente drena una microcuenca tributaria del río Tacotalpa y forma parte de la cuenca Grijalva-Villahermosa en su porción media (Instituto Nacional de Estadística, Geografía e Historia, 2000). La microcuenca se sitúa en la región ecogeográfica laderas septentrionales de la Sierra Norte de Chiapas, entre 40 m y 620 m sobre el nivel del mar, en relieves dominantes de laderas escarpadas y cimas sobre rocas calizas-lutitas del Cretácico y algunas áreas onduladas a planas sobre lutitas-areniscas del Terciario Paleoceno y sedimentos aluviales del Cuaternario Holoceno, en la zona baja (Ortiz-Pérez, Siebe y Cram, 2005; SGM, 2005). El clima es cálido húmedo con lluvias todo el año $\mathrm{Af}(\mathrm{m})$, tiene temperatura media anual de $25.6{ }^{\circ} \mathrm{C}$ y precipitación media anual de 3458.1 mm (García, 2004). En la vegetación ribereña se han documentado las especies Inga vera, Lonchocarpus hondurensis, Spondias mombin, Tabebuia rosea; y en las zonas posteriores crecen especies de selva alta perennifolia como Brosimum alicastrum, Dialium guianense, Nectandra sp. y Cordia alliodora (Pérez, Sousa, Hanan, Chiang y Tenorio, 2005). 

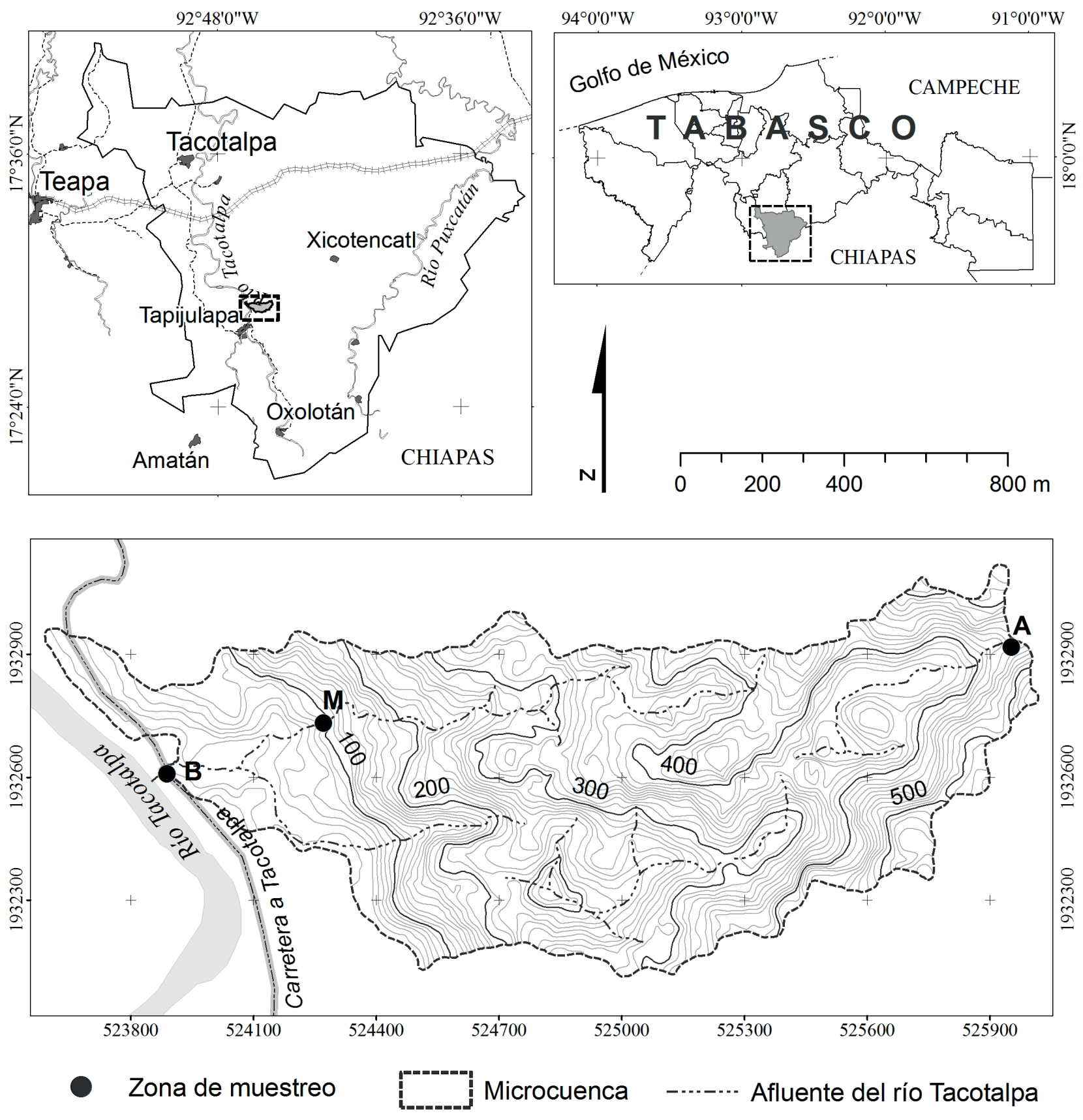

Figura 1. Ubicación geográfica de las zonas de estudio en un afluente del río Tacotalpa, Tabasco.

\section{Zonas de muestreo}

Siguiendo el perfil longitudinal del afluente, en forma ascendente, se ubicaron tres zonas de muestreo en las siguientes coordenadas: Baja (B) a $17^{\circ} 28^{\prime} 46^{\prime \prime} \mathrm{N}$ y $92^{\circ} 46^{\prime}$ 30 " W; Media (M) a $17^{\circ} 28^{\prime} 50^{\prime \prime} \mathrm{N}$ y $92^{\circ} 46^{\prime} 17^{\prime \prime}$ W y Alta (A) a $17^{\circ} 28^{\prime} 56^{\prime \prime} \mathrm{N}$ y $92^{\circ} 45^{\prime} 20^{\prime \prime}$ W con altitudes de
41 m, 125 m y 464 m snm (Fig. 1). La zona B se localizó en una terraza sobre sedimentos aluviales del Cuaternario Holoceno y colinas sobre lutitas-areniscas del Terciario Paleógeno Paleoceno, la pendiente varía de $6 \%$ a $25 \%$ y el suelo varió de Fluvisol Mólico, profundo y fértil, a Acrisol Cutánico, profundo y de baja fertilidad. Las zonas M y A 
se localizaron en laderas inclinadas y escarpadas sobre rocas calizas-lutitas del Cretácico Superior, con pendientes de $40 \%$ a $100 \%$; el suelo fue un Leptosol Réndzico, delgado, con buen contenido nutrimental; la alta precipitación pluvial y las pendientes propician los procesos de intemperización, kastificación y erosión (SGM, 2005; Zavala-Cruz et al., 2011; Zavala-Cruz et al., 2016).

\section{Muestreo de vegetación ribereña}

Se realizó por el método de transecto. En cada zona se establecieron ocho unidades de muestreo de $5 \mathrm{~m} \times 50 \mathrm{~m}$ $\left(2000 \mathrm{~m}^{2}\right)$, cuatro se ubicaron en cada lado del afluente, mediando una distancia de $5 \mathrm{~m}$ entre las unidades. Se censaron todos los árboles y arbustos con DAP $\geq 1 \mathrm{~cm}$ (Zarco-Espinosa, Valdez-Hernández, Ángeles-Pérez y Castillo-Acosta, 2010). La identificación de especies se apoyó en bibliografía especializada (Pennington y Sarukhán, 2005; Pérez et al., 2005), y comparación con ejemplares de los herbarios virtuales MBG Trópicos (http:// www.tropicos.org/), y Muestras Neotropicales de Herbario (http://fm1.fieldmuseum.org/vrrc/index.php). Algunos ejemplares solamente se identificaron a nivel de familia, debido a que carecían de estructuras reproductivas.

\section{Índice del valor de importancia (IVI)}

Se determinó mediante la suma de los valores relativos de densidad, frecuencia y dominancia (Smith y Smith, 2007).

\section{Diversidad de especies}

Se calculó con el índice de Shannon-Wiener:

$H^{\prime}=-\Sigma p i * \ln p i$

donde: $p i=$ abundancia proporcional de la especie $i$; para ello, se calculó $p i=n i / \mathrm{N}$, que es el número de individuos de una especie entre el número de individuos de todas las especies (Moreno, 2001). La diferencia estadística, se determinó mediante el método de Hutcheson (Magurran y McGill, 2011) para calcular el valor de $t$ modificado:

$t=\left(H^{\prime} 1-H^{\prime} 2 / \operatorname{VarH}^{\prime} 1-\operatorname{Var} H^{\prime} 2\right)^{1 / 2}$ donde: $H^{\prime} 1$ = diversidad de comunidad A; $H^{\prime} 2$ = diversidad de comunidad B; VarH'1 = varianza de diversidad de comunidad A, y VarH'2 = varianza de diversidad de comunidad B.

Los grados de libertad $(g l)$ se determinaron con la ecuación:

$g l=\left(\operatorname{VarH} H^{\prime} 1+\operatorname{VarH}^{\prime} 2\right)^{2} /\left[\left(\operatorname{Var} H^{\prime} 1\right)^{2} / N 1\right]+\left[\left(\operatorname{VarH} H^{\prime} 2\right)^{2} / N 2\right]$

La varianza de $H^{\prime}$ de cada zona se determinó con la ecuación:

$\operatorname{VarH} H^{\prime}=\left[\sum p i(\ln p i)^{2}-\left(\sum p i \ln p i\right)^{2} / N\right]-\left[S-1 / 2 N^{2}\right]$

\section{Índice de Similitud}

Se calculó con el índice de Sorensen:

$(I S)=2 C / A+B * 100$

donde: $A=$ número de especies en comunidad $\mathrm{A} ; B=$ número de especies en comunidad $\mathrm{B}, \mathrm{y} C=$ número de especies comunes en ambas comunidades (Moreno, 2001).

\section{Muestreo y análisis de suelo}

En cada unidad de muestreo de vegetación se obtuvo una muestra de suelo de $500 \mathrm{~g}$ a una profundidad de $0 \mathrm{~cm}$ a 30 cm (Salgado-García, Palma-López, Castelán-Estrada, Lagunes-Espinoza y Ortiz, 2013). En el laboratorio se determinaron las propiedades de acuerdo con la NOM021-RECNAT-2000 (Diario Oficial de la Federación, 2002): materia orgánica (MO) por el método de Walkley y Black, $\mathrm{pH}$ mediante potenciometría en agua (relación 1:2), textura (higrómetro) por el método de Bouyoucos, nitrógeno total $(\mathrm{Nt})$ por procedimientos de digestión, fósforo $(\mathrm{P})$ extractable por el procedimiento de Olsen, Potasio $(\mathrm{K})$ empleando acetato de amonio $1 \mathrm{~N}, \mathrm{pH} 7.0$ como solución extractante. La interpretación de los datos físicos y químicos de suelos se basó en criterios establecidos en la NOM-021-RECNAT-2000 (Diario Oficial de la Federación, 2002) y Salgado-García et al. (2013). 


\section{Análisis estadístico}

Se realizó una comparación de medias de Tukey $(\alpha=$ 0.05 ) con las variables de vegetación ribereña (abundancia, riqueza y altura) y suelo $(\mathrm{pH}, \mathrm{Nt}, \mathrm{MO}, \mathrm{P}, \mathrm{K}$, arcilla, limo y arena), para conocer si existen diferencias significativas entre las zonas de muestreo; y una correlación de Pearson para determinar la asociación entre variables de vegetación y suelo. El análisis se hizo con el programa estadístico SPSS (Castañeda, Cabrera, Navarro y Vries, 2010). Se complementó con el análisis de componentes principales (ACP) con el programa PAST (Hammer, 2012).

\section{Resultados}

\section{Composición florística}

Se registraron 484, 685 y 512 individuos en las zonas B, M y A, respectivamente, con un total de 1681; distribuidos en 131 especies, la zona A presentó mayor número (82); y 38 familias botánicas, la zona M mostró una mayor cantidad (35) (Tabla 1). Las familias con mayor número de especies fueron Fabaceae (21), Euphorbiaceae (12) y Moraceae (10) (Anexo 1).

\section{Estructura vertical}

Se encontraron tres estratos del bosque ribereño a lo largo del afluente, inferior $(0 \mathrm{~m}-10 \mathrm{~m})$, medio $(10 \mathrm{~m}-20 \mathrm{~m}) \mathrm{y}$ superior (20 m - 30 m o más). El estrato inferior domina en las tres zonas, con mayor cantidad de individuos en la zona $\mathrm{M}$ (678). Los estratos medio y superior tienen individuos solamente en las zonas $\mathrm{M}$ y $\mathrm{A}$, sobresaliendo la

Tabla 1. Composición florística de vegetación ribereña en tres zonas de un afluente del río Tacotalpa, Tabasco.

\begin{tabular}{cccc}
\hline Zona & Número de individuos & Especie & Familia \\
\hline B & 484 & 56 & 19 \\
M & 685 & 72 & 35 \\
A & 512 & 82 & 27 \\
Total & 1681 & 131 & 38 \\
\hline
\end{tabular}

última (49); mientras que la zona B carece prácticamente de los estratos inferior y medio (Fig. 2).

\section{Estructura horizontal}

Las tres zonas del afluente mostraron mayor concentración de individuos en las primeras categorías diamétricas y pobre representación en diámetros mayores; en B no se registraron individuos en la cuarta, quinta y sexta categorías; en $\mathrm{M}$ solo se registraron individuos en las categorías primera, segunda, tercera y quinta, y en A en todas las categorías excepto la quinta (Fig. 3). El promedio del área

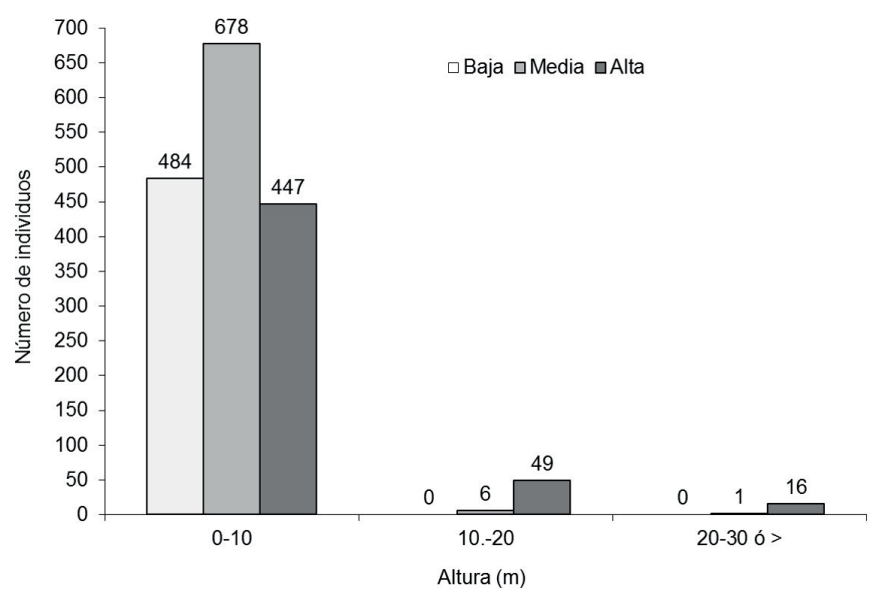

FigURA 2. Estratificación vertical de vegetación ribereña en tres zonas de un afluente del río Tacotalpa.

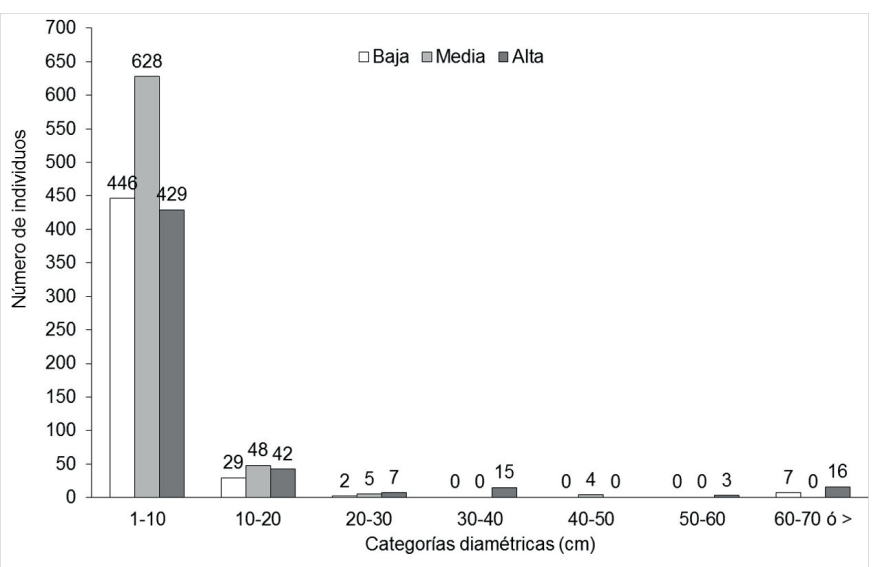

Figura 3. Distribución diamétrica de vegetación ribereña en tres zonas de un afluente del río Tacotalpa. 
basal de la zona B fue $469.02 \mathrm{~m}^{2} \pm 1265.01 \mathrm{~m}^{2}(0.47 \mathrm{ha})$, en la zona $\mathrm{M}$ fue $83.98 \mathrm{~m}^{2} \pm 87.96 \mathrm{~m}^{2}(0.01 \mathrm{ha})$ y 614.55 $\mathrm{m}^{2} \pm 597.70 \mathrm{~m}^{2}$ (0.06 ha) en A.

\section{Índice de valor de importancia (IVI)}

Por zona, el IVI en B varió de 11.8 a 59.9, sobresaliendo Ficus yoponensis; en $\mathrm{M}$ las especies tuvieron un índice menor a 26.6 y en A osciló entre 12.5 y 39.9; Mortoniodendron anisophyllum fue la especie más importante en las últimas dos zonas. Las especies restantes estuvieron debajo de estos valores. La zona B registró una especie arbustiva de la familia Fabaceae y cuatro especies arbóreas; $\mathrm{M}$ dos especies arbustivas: Belotia sp. y $P$. auritum y tres especies arbóreas y A solamente una especie arbustiva: Acalipha sp.2 y cuatro arbóreas (Tabla 2).

\section{Diversidad de especies}

La diversidad de especies vegetales fue similar entre las tres zonas (3.27 en B; 3.54 en M y 3.81 en A). Los valores promedio de diversidad y equitatividad fueron ligeramente mayores en $\mathrm{A}$, excepto la riqueza que fue mayor en $\mathrm{M}$; pero la desviación estándar es el doble en M (mayor variabilidad) respecto a las zonas B y A (Tabla 3).

\section{Similitud florística}

La similitud fue ligeramente mayor en las zonas M-A (53.25\%), seguido de M-B (53.13\%) y B-A (31.88\%). La zona $\mathrm{B}$, confirma que la curva de acumulación de especies se encuentra por debajo de M y A (Fig. 4).

\section{Variables de vegetación ribereña y suelo}

La altura de los árboles ribereños de la zona A mostró diferencia significativa respecto a las zonas M y B (Fig. 5). En la zona M los valores más elevados de arcilla y limo determinaron la textura franco arcillosa, siendo estadísticamente diferentes respecto a las zonas A y B; en estas zonas, el mayor contenido de arena condicionó la textura franco arenosa. El pH mostró diferencias entre zonas, variando de medianamente alcalino en $\mathrm{A}$ y $\mathrm{B}$ a neutro en M. Los contenidos de $\mathrm{Nt}, \mathrm{MO}$ y $\mathrm{P}$ de la zona A fueron estadísticamente diferentes respecto a las zonas B y $\mathrm{M}$; y los valores en A superaron de 2.5 a 5.3 veces a los de B. Los niveles de $\mathrm{K}$ fueron similares en las tres zonas (Tabla 4 y Fig. 5). Las variables químicas $\mathrm{Nt}$ y $\mathrm{MO}$ variaron de ricas en $\mathrm{A}$ a pobres en $\mathrm{B}$; las de $\mathrm{P}$ y $\mathrm{K}$ fueron bajas en todas las zonas (Tabla 4).

\section{Relación entre las variables de vegetación ribereña y suelo}

Los coeficientes de correlación de Pearson muestran que la abundancia de la vegetación riparia se asoció positivamente con el limo y negativamente con el pH y la arena; además, la altura de los árboles se correlacionó positivamente con las variables $\mathrm{Nt}, \mathrm{MO}$ y $\mathrm{P}$ y negativamente con el limo. Es evidente que el mayor contenido de $\mathrm{MO}$ y de las

TABla 2. Especies con mayor Índice de Valor de Importancia (IVI) en las tres zonas de un afluente del río Tacotalpa, Tabasco.

\begin{tabular}{|c|c|c|c|c|c|c|c|c|c|c|c|c|c|c|}
\hline \multicolumn{5}{|c|}{ Baja } & \multicolumn{5}{|c|}{ Media } & \multicolumn{5}{|c|}{ Alta } \\
\hline Especies & $\mathrm{Dr}$ & $\mathrm{Fr}$ & Dor & $\mathrm{IVI}$ & Especies & Dr & $\mathrm{Fr}$ & Dor & IVI & Especies & $\mathrm{Dr}$ & $\mathrm{Fr}$ & Dor & IVI \\
\hline Ficus yoponensis & 1.2 & 2.1 & 56.5 & 59.9 & M. anisophyllum & 0.6 & 1.0 & 25.4 & 26.6 & M. anisophyllum & 0.1 & 1.6 & 37.4 & 39.9 \\
\hline Citrus reticulata & 0.2 & 0.7 & 33.3 & 34.2 & Piper auritum & 12.3 & 2.5 & 0.2 & 14.9 & Brosimum guianense & 2.0 & 3.1 & 13.2 & 18.2 \\
\hline Fabaceae (NI) & 12.6 & 5.7 & 0.1 & 18.3 & Gliricidia sepium & 0.2 & 0.5 & 13.2 & 13.8 & Pterocarpus rohrii & 0.2 & 0.5 & 14.2 & 14.9 \\
\hline Hasseltia mexicana & 10.5 & 3.6 & 0.1 & 14.2 & Hampea nutricia & 0.2 & 0.5 & 11.9 & 12.4 & Acalypha sp.2 & 12.5 & 2.1 & 0.0 & 14.6 \\
\hline Trichilia havanensis & 6.0 & 5.0 & 0.8 & 11.8 & Belotia sp. & 8.3 & 2.5 & 0.1 & 10.8 & Recchia simplicifolia & 9.4 & 3.1 & 0.1 & 12.5 \\
\hline
\end{tabular}

$\mathrm{NI}=$ no identificado, $\mathrm{Dr}=$ Densidad relativa, $\mathrm{Fr}=$ Frecuencia relativa, Dor $=$ Dominancia relativa 
Tabla 3. Valores promedio de riqueza (S), diversidad $\left(\mathrm{H}^{\prime}\right)$ y equitatividad (E) en tres zonas de un afluente del río Tacotalpa, Tabasco.

\begin{tabular}{cccc}
\hline Zona & $\mathrm{S}$ & $\mathrm{H}^{\prime}$ & $\mathrm{E}$ \\
\hline $\mathrm{B}$ & $19.38 \pm 4.98$ & $2.48 \pm 0.39$ & $0.85 \pm 0.05$ \\
M & $26.63 \pm 9.88$ & $2.69 \pm 0.67$ & $0.83 \pm 0.12$ \\
A & $25.38 \pm 5.26$ & $2.88 \pm 0.34$ & $0.90 \pm 0.07$ \\
\hline
\end{tabular}

variables de fertilidad del suelo, incrementan la altura de los árboles, igualmente, el mayor contenido de limo en la zona $\mathrm{M}$ contribuye a la abundancia de árboles (Tabla 5).

El orden de datos en el ACP se tomó de los primeros tres componentes que tuvieron valores propios mayores a 1 , los cuales explican $80.16 \%$ de la variación, siendo una proporción significativa ya que solo se pierde $19.84 \%$ de la varianza (Tabla 6). En la figura 6 se muestra la disposición espacial de las unidades muestrales sobre el plano definido por los dos primeros ejes que en conjunto representan $62.13 \%$ de la varianza total de los datos. Las variables altura $\mathrm{MO}, \mathrm{Nt}, \mathrm{P}$ y $\mathrm{K}$ se correlacionaron positivamente y significativamente con el primer componente, donde $\mathrm{Nt}$ y $\mathrm{MO}$ tuvieron un valor mayor a 0.45 ; mientras que en el segundo componente las variables abundancia, limo y arcilla representaron mayor contribución positiva (Tabla 6 y Fig. 6).

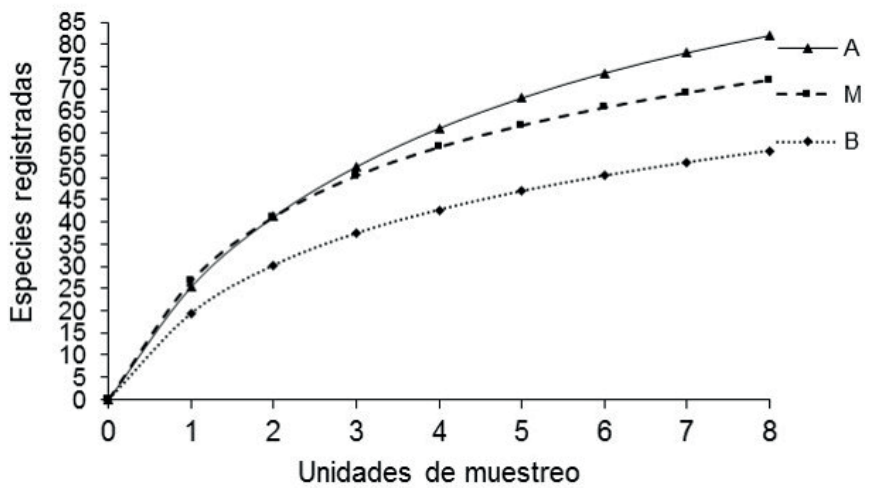

Figura 4. Curva de especies-área en tres zonas de muestreo en un afluente del río Tacotalpa.

\section{DISCUSIÓN}

\section{Composición florística}

La riqueza específica del afluente del río Tacotalpa fue alta (131 especies), superando lo documentado en otros estudios de vegetación ribereña en zonas tropicales (Rodríguez y Colonnelo, 2009; Sirombra y Mesa, 2010). Las familias Fabaceae, Euphorbiacea y Moraceae, también son señaladas por Maldonado-Sánchez y MaldonadoMares (2010) y Vásquez-Negrín, Castillo-Acosta, ValdezHernández, Zavala-Cruz y Martínez-Sánchez (2011) como las más representativas por el mayor número de

Tabla 4. Valores promedio de propiedades del suelo en la capa de $0 \mathrm{~cm}-30 \mathrm{~cm}$ de profundidad en tres zonas de un afluente de río Tacotalpa, Tabasco.

\begin{tabular}{|c|c|c|c|c|c|c|c|c|c|c|}
\hline \multirow[t]{2}{*}{ Zona } & & h & $\mathrm{Nt}$ & MO & \multirow{2}{*}{$\begin{array}{c}\mathrm{P} \\
\mathrm{mg} \mathrm{kg}{ }^{-1}\end{array}$} & \multirow{2}{*}{$\begin{array}{c}\mathrm{K} \\
\mathrm{cmol} \mathrm{kg}^{-1}\end{array}$} & Arc & $\mathrm{Li}$ & Are & \multirow{2}{*}{$\begin{array}{c}\text { Clasificación } \\
\text { Textural }\end{array}$} \\
\hline & \multicolumn{2}{|c|}{ rel. 1:2 } & \multicolumn{2}{|c|}{$\%$} & & & & $\%$ & & \\
\hline \multirow[t]{2}{*}{ B } & \pm & 7.81 & 0.06 & 1.06 & 1.66 & 0.18 & 15.5 & 26.88 & 57.63 & Franco \\
\hline & D.E & 0.22 & 0.03 & 0.44 & 0.31 & 0.06 & 2.78 & 6.42 & 8.63 & arenoso \\
\hline \multirow[t]{2}{*}{$M$} & \pm & 6.82 & 0.16 & 2.66 & 2.41 & 0.20 & 31.5 & 38.75 & 29.75 & Franco \\
\hline & D.E & 0.81 & 0.02 & 0.53 & 0.73 & 0.06 & 3.82 & 2.87 & 4.43 & arcilloso \\
\hline \multirow[t]{2}{*}{$A$} & \pm & 7.47 & 0.32 & 5.70 & 4.20 & 0.24 & 25.25 & 18.75 & 56.13 & Franco \\
\hline & D.E & 0.18 & 0.11 & 2.12 & 1.15 & 0.06 & 20.31 & 5.04 & 16.3 & arenoso \\
\hline
\end{tabular}



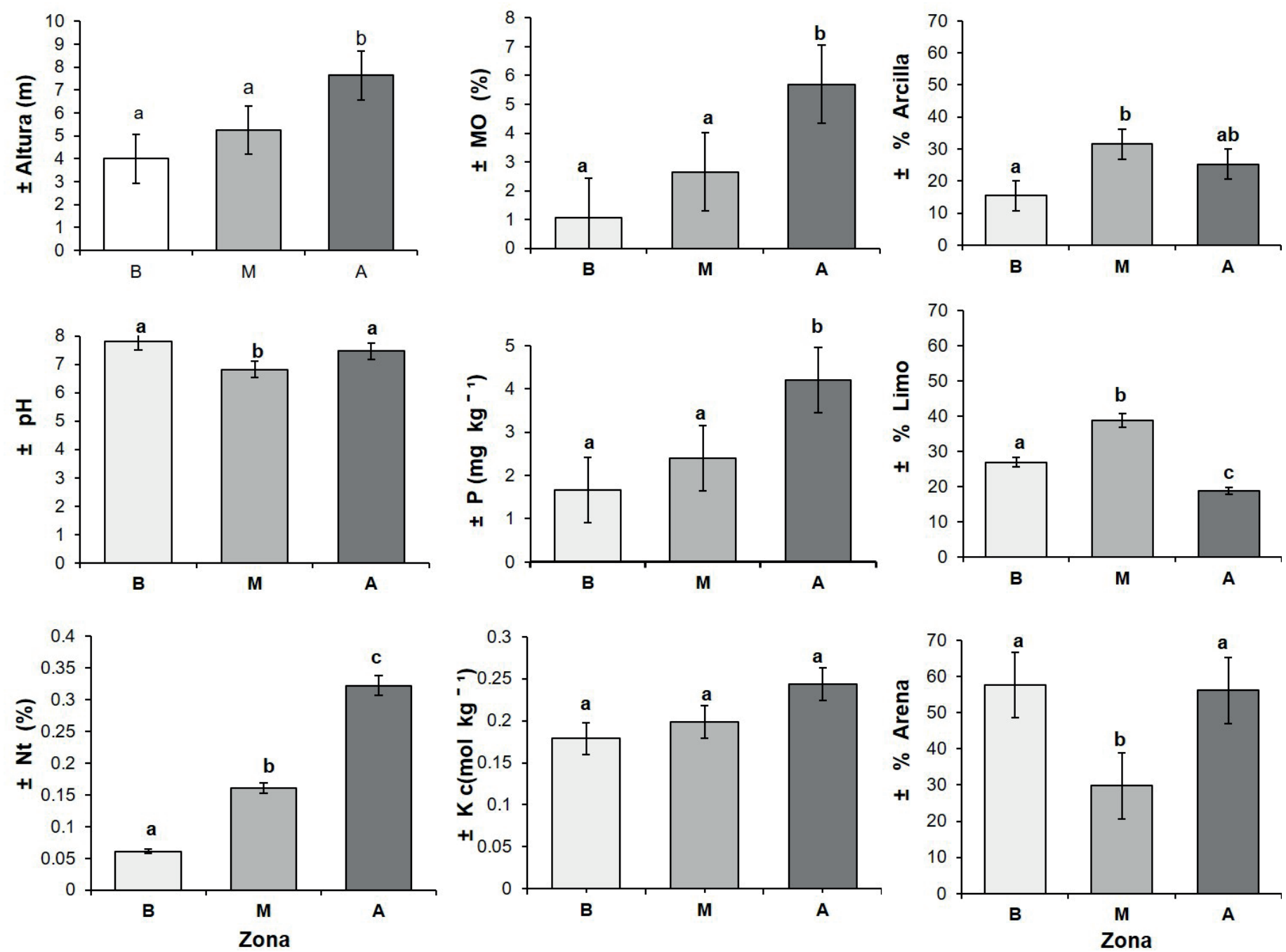

Figura 5. Comparación de medias de variables de vegetación ribereña y suelo en tres zonas de un afluente del río Tacotalpa $(\mathrm{p}<0.05)$. Las barras indican la desviación estándar.

TABLA 5. Correlación de Pearson entre variables de vegetación ribereña con las propiedades del suelo de un afluente del río Tacotalpa, Tabasco.

\begin{tabular}{cccccccccc}
\hline Variables & $\mathrm{pH}$ & $\mathrm{Nt}$ & $\mathrm{MO}$ & $\mathrm{P}$ & $\mathrm{K}$ & $\mathrm{Arc}$ & $\mathrm{Li}$ & Are \\
\hline \multirow{2}{*}{ Abundancia } & $-0.54^{* *}$ & 0.20 & 0.19 & 0.14 & -0.29 & 0.37 & $0.44^{*}$ & $-0.55^{* *}$ \\
& 0.01 & 0.34 & 0.38 & 0.52 & 0.17 & 0.08 & 0.03 & 0.01 \\
\multirow{2}{*}{ Riqueza } & -0.05 & 0.28 & 0.28 & 0.35 & -0.08 & 0.02 & 0.10 & -0.07 \\
& 0.84 & 0.17 & 0.19 & 0.10 & 0.70 & 0.93 & 0.65 & 0.75 \\
\multirow{2}{*}{ Altura } & 0.02 & $0.73^{* *}$ & $0.73^{* *}$ & $0.66^{* *}$ & 0.25 & 0.22 & $-0.52^{* *}$ & 0.13 \\
& 0.94 & 0.00 & 0.00 & 0.00 & 0.25 & 0.30 & 0.01 & 0.55 \\
\hline
\end{tabular}




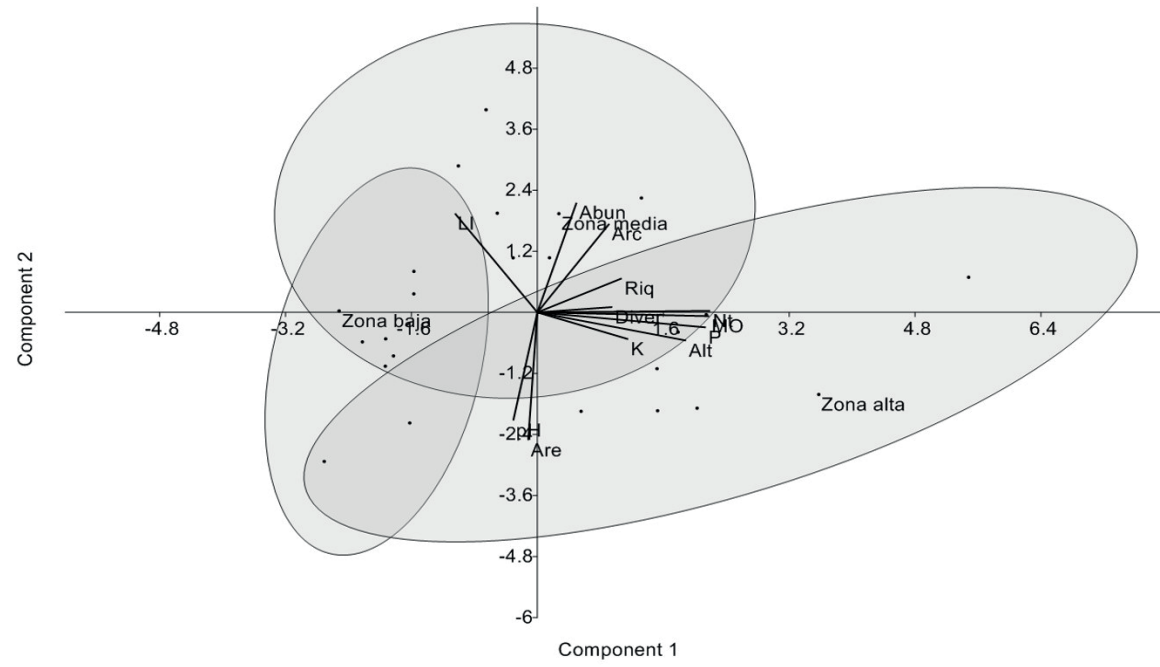

Figura 6. Análisis de componentes principales de las variables de vegetación ribereña y suelo en tres zonas de un afluente del río Tacotalpa. Los puntos corresponden a las unidades muestrales.

Tabla 6. Coeficientes de correlación de los componentes principales de las variables de vegetación ribereña y suelo.

\begin{tabular}{lccc}
\hline ACP & CP 1 & CP 2 & CP 3 \\
\hline Eigenvalues & 4.361 & 3.094 & 2.163 \\
\% de varianza explicada & 36.343 & 25.783 & 18.029 \\
\hline Abundancia (Abun) & 0.103 & 0.446 & 0.239 \\
Riqueza (Riq) & 0.222 & 0.138 & 0.529 \\
Diversidad (Diver) & 0.197 & 0.022 & 0.575 \\
Altura (Alt) & 0.390 & -0.114 & 0.063 \\
Potencial de Hidrógeno (pH) & -0.063 & -0.439 & 0.152 \\
Nitrógeno total (Nt) & 0.454 & 0.006 & -0.073 \\
Materia orgánica (MO) & 0.451 & -0.015 & -0.078 \\
Fósforo disponible (P) & 0.442 & -0.061 & -0.054 \\
Potasio (K) & 0.239 & -0.109 & -0.395 \\
Arcilla (Arc) & 0.189 & 0.360 & -0.303 \\
Limo (Li) & -0.216 & 0.402 & 0.099 \\
Arena (Are) & -0.024 & -0.519 & 0.185 \\
\hline
\end{tabular}

especies en la zona de estudio y por su mayor distribución en los bosques tropicales. De acuerdo con Ceccon (2003), estas características indican que el afluente aún mantiene la riqueza de especies vegetales. En la zona $\mathrm{B}$, cerca de la confluencia con el río Tacotalpa, el afluente evidenció menor riqueza, debido posiblemente a la incidencia de inundaciones temporales por ubicarse en una posición topográfica baja y receptora del caudal, como lo sustentan Moffat, McLachlan y Kenkel. (2004). También puede deberse al impacto de las actividades humanas (Yang, Dilts, Condon, Turner y Weisberg, 2011). Las zonas M y A presentaron mayor riqueza de especies correspondientes a remanentes de vegetación menos alterada (Mingxi, Hongbing, Oinghua y Gang, 2005).

\section{Estructura vertical y horizontal de vegetación ribereña}

La estructura vertical del bosque ribereño se concentró en el estrato bajo con $95.72 \%$, coincidiendo con los registros de Acosta et al. (2008), Vásquez et al. (2011) y LópezPérez, Castillo-Acosta, Zavala-Cruz y Hernández-Trejo (2014). La zona B registró alturas menores a $10 \mathrm{~m}$, de las cuales $36 \%$ son especies arbustivas y $64 \%$ arbóreas; $M$ presentó $26 \%$ arbustivas y $74 \%$ arbóreas, mientras que A exhibió $24 \%$ arbustivas y $76 \%$ arbóreas. Estos datos sugieren que existen diferentes estados de recuperación en las tres zonas. Al respecto, Kaneko y Takada (2008) men- 
cionan que los patrones de especies arbóreas menores a 10 $\mathrm{m}$ de altura, como ocurre en la zona B, pueden estar siendo provocados por topografía aluvial que crea diferentes patrones de espacios en los que la altura de los árboles no rebasa este límite. Las zonas M y B presentaron abundancia de las especies Acalypha sp., A. angustissima y del género Piper con alturas menores a $5 \mathrm{~m}$, siendo indicadoras de vegetación secundaria de crecimiento rápido, que aprovechan los claros provocados por las actividades humanas (Martínez-Ramos y García-Orth, 2007). La zona A presentó las especies Acalypha sp2. y Recchia simplicifolia como las más abundantes; la última es indicadora de la presencia de especies plehistocénicas (Wendt y Lott, 1985).

La distribución horizontal indica alta concentración de individuos en diámetros menores de $10 \mathrm{~cm}(94.65 \%)$ en concordancia con los registros de Flores, Batte y Dapara (2002). Los individuos de diámetros pequeños forman parte de un proceso de sucesión ecológica, donde pocos individuos sobreviven y alcanzan el tamaño necesario conforme avanza la regeneración en un espacio y tiempo (Martínez-Ramos y García-Orth, 2007), de ahí la importancia de la existencia de individuos abundantes en este estrato (Zarco et al., 2010). La zona B presentó buena regeneración pero reclutamiento discontinuo; $\mathrm{M}$ con buena regeneración y reclutamiento continuo hasta la tercera categoría, y A exhibió buena regeneración y reclutamiento continuo. La discontinuidad de los individuos en las zonas B y $\mathrm{M}$ es evidencia del disturbio ocasionado por actividades humanas como la extracción de leña y la agricultura, lo que ha provocado variaciones espaciales en la estructura y evolución de las comunidades vegetales (Fernández-Aláez, Fernández-Aláez y García-Criado, 2005; Kaneko y Takada, 2008). También, el abandono de las parcelas de cultivo en las riberas adyacentes contribuye a la recuperación de la vegetación secundaria, produciendo notables cambios fisonómicos en el bosque ribereño (Aguirre, Cabrera, Sánchez, Merino y Maza, 2003; Sirombra y Mesa, 2010). El promedio del área basal en M confirma que se encuentra en proceso de regeneración más temprana con respecto a las dos zonas B y A.

\section{Índice de valor de importancia (IVI)}

El alto IVI de las especies destaca aquellas que están mejor adaptadas por su dominancia, abundancia o distribución a lo largo del afluente como lo mencionan Acosta et al. (2008). En el afluente en estudio, F. yoponensis, M. anishophyllum, H. nutricia, B. guianense y $P$. rohrii fueron las especies arbóreas más importantes por su mayor diámetro; H. mexicana, P. auritum y R. simplicifolia destacaron por ser muy abundantes. La abundancia de algunas especies arbustivas en las zonas B y $\mathrm{M}$ reafirma el grado de alteración que ha sufrido el afluente, ya que estas especies se establecen comúnmente en lugares alterados (Acosta et al., 2008). La zona A, registró cuatro especies arbóreas más importantes, debido a que se encuentra aledaña a vegetación natural (Mingxi et al., 2005).

\section{Diversidad de especies}

La diversidad de especies fue similar, debido a que cada zona registró abundancia de una o dos especies que no se comparten (Camacho et al., 2006). Por ejemplo, A. angustissima solo se registró en $\mathrm{B}$, Acalypha sp.1 en $\mathrm{M}$ y $R$. simplicifolia en A, lo cual contribuye a homogenizar los valores del índice. La equitatividad fue mayor a 80 en las tres zonas, indicando que la abundancia de las especies se distribuye de manera uniforme. La diversidad fue alta superando lo encontrado en otros estudios (Treviño, Cavazos y Aguirre, 2001; Sunil et al., 2010). La diversidad florística arbórea en las tres zonas puede deberse a alteración por actividades humanas, además es producto del flujo natural de los sedimentos a lo largo del bosque ribereño (Johnson, 2002); estas zonas funcionan como corredores para la dispersión de diferentes especies de plantas, desde las partes altas de laderas hacia las partes bajas creando metacomunidades (Araujo et al., 2013).

\section{Similitud florística}

De las 131 especies registradas, 19 (14.50\%) se compartieron entre las tres zonas. El mayor número de especies compartidas se presentó entre $\mathrm{M}$ y A con 41, seguido de $\mathrm{B}$ y $\mathrm{M}$ con 34 y, por último, B y A con 22. Araujo et al. (2013) mencionan que zonas con baja similitud se deben a 
un aislamiento; mientras que a mayor similitud mejor conectividad con remanentes de vegetación natural, como es el caso de las zonas M y A (41). La curva de acumulación de especies no mostró clara tendencia asintótica, pero las especies que faltaron por encontrar son probablemente especies localmente raras, o individuos erráticos en fase de dispersión procedentes de poblaciones estables externas al afluente estudiado (Moreno y Halffter, 2001).

\section{Variables de vegetación ribereña y suelo}

Respecto a la diferencia de altura de los árboles entre zonas, en A se debe a la presencia de especies de vegetación original (como Ocotea sp., B. guianense y P. rohrii) y especies de vegetación secundaria que superan los $20 \mathrm{~m}$ de altura por su mayor tiempo de recuperación (como $S$. mombin, C. elástica, B. simaruba, C. obtusifolia y L. hondurensis). Estas especies son indicadoras de mayor recuperación de la vegetación (Pennington y Sarukhán, 2005; Pérez et al., 2005). La presencia de Q. funebris en $\mathrm{M}$ indica que se encuentra en proceso de sucesión temprana funcionando como sitios de reservas de especies nativas para etapas más tardías (Ochoa-Gaona, Hernández-Vázquez, De Jong y Gurri-García, 2007).

$\mathrm{El} \mathrm{pH}$ del suelo, moderadamente alcalino, se explica por la presencia de rocas calizas en la zona A, y en B por la acumulación de sedimentos ricos de $\mathrm{Ca}$ aportados por las corrientes del afluente y su microcuenca (Zavala-Cruz et al., 2011; Kamrani et al., 2011); el pH neutro en M corresponde a un suelo desarrollado sobre roca madre de lutitas con menor contenido de Ca (SGM, 2005).

La mayor concentración de $\mathrm{MO}$ y $\mathrm{Nt}$ en A se relaciona con la vegetación más conservada del afluente. Este resultado concuerda con Sepúlveda et al. (2009) y Murray et al. (2011) quienes mencionan que la MO está influenciada principalmente por el estado de conservación de la vegetación, al acumularse mayor cantidad de residuos vegetales de árboles más altos, el contenido de MO incrementa en el suelo y, a su vez, influye en el alto contenido de $\mathrm{Nt}$ (Alvares, 2001). En la zona B los contenidos bajos de Nt y MO se asocian a la vegetación secundaria con menos tiempo de recuperación después del abandono de parcelas de cultivo (Álvarez, Ibáñez, García y Almendros, 2012), lo que se traduce en vegetación poco desarrollada que aporta menos hojarasca al suelo (Sánchez, Ruíz y Ruíz, 2005; García y Vollmann, 2012). En la zona B el bajo contenido de MO influye en los menores niveles de $\mathrm{Nt}$, P y K, similar a lo encontrado por otros autores (Porta, López y Roquero, 2003; Palma-López, Cisneros, Moreno y Rincón-Ramírez, 2007). Además, el P y K son nutrientes móviles que declinan rápidamente al inicio de la descomposición de MO (Alvares, 2001), proceso que ocurre después de la tala de vegetación primaria en las zonas tropicales (Paul, 2007). Por consiguiente, en la zona A, los mayores contenidos de $\mathrm{MO}$ y nutrimentos en el suelo generan condiciones más favorables para el desarrollo de la vegetación riparia.

\section{Relación de vegetación ribereña y suelo}

La relación positiva entre la abundancia de las plantas leñosas y el limo, indica que esta variable contribuye a la mayor cantidad de árboles y arbustos ribereños; sin embargo, más que la fracción limosa, la mayor abundancia en B parece explicarse por la presencia de especies de vegetación secundaria como $P$. auritum, $P$. arboreum, $H$. mexicana, Acalypha sp.1, Belotia sp., V. patens, A. angustissima y L. hondurensis (Pérez et al., 2005). Inversamente, las variables $\mathrm{pH}$ y arena mostraron asociación negativa con la abundancia ( $\mathrm{p} \leq 0.01$ ), indicando que al disminuir la alcalinidad y el porcentaje de arena en el suelo, la abundancia aumenta, como ocurrió en la zona $\mathrm{M}$ con $\mathrm{pH}$ neutro, buen porcentaje de arcilla y moderado a bajo porcentaje de arena. La riqueza de especies no se correlacionó con las variables edáficas, se infiere que la riqueza del afluente depende de otros factores como pueden ser la heterogeneidad del relieve, los gradientes microclimáticos y los microambientes disponibles para el establecimiento de especies (Smith y Smith, 2007).

La altura de los árboles ribereños se correlacionó positivamente con las variables edáficas MO, Nt y P, indicando que la altura se incrementa conforme mejoran estas propiedades del suelo. Se ha demostrado que la MO es la fuente principal del $\mathrm{N}$ y como un nutrimento esencial para el cre- 
cimiento de las plantas (Gallardo, Covelo, Morillas y Delgado, 2009). Las especies con altura mayor a $20 \mathrm{~m}$ se registraron en A, confirmando que esta zona tiene las mejores características edáficas para el desarrollo de los árboles; estos, a la vez, contribuyen con materiales orgánicos que al transformarse en $\mathrm{MO}$, a través del proceso de mineralización, liberan compuestos inorgánicos como $\mathrm{NO}_{4}, \mathrm{NH}_{4}^{+}, \mathrm{P}$ y K que son absorbidos por las raíces de las plantas (Sánchez et al., 2005). También se observó correlación negativa del contenido de limo con la altura de los árboles, indicando que la altura disminuye al aumentar la cantidad de limo; esta situación se presentó en las zonas B y M, coincidiendo con los mayores porcentajes de limo en el afluente (26.8\% y $38.7 \%$ ) y los árboles de menor altura. El ordenamiento de los datos en ACP permite deducir que las variables riqueza, diversidad, altura, Nt, MO, P y K tienden a incrementarse hacia la zona alta; mientras que las variables abundancia, arcilla y limo exhiben una mayor tendencia hacia la zona media; en tanto que el $\mathrm{pH}$ junto con la arena se reducen en la zona media y tienden a aumentar hacia la zona B (Fig. 6); debido a que en la zona topográfica baja del afluente se acumula materiales aluviales y cationes como el calcio (Chun, Zueng y Chang, 2004; Yimer, Ledin, y Abdelkadir, 2006). Este análisis demuestra que la interacción negativa y positiva de algunas variables de la vegetación ribereña con las propiedades edáficas, es dinámica y compleja y es reconocida por su importancia en el funcionamiento de estos ecosistemas (Aponte, 2011).

\section{CONCLUSIONES}

En tres zonas de vegetación ribereña de un afluente del río Tacotalpa, se registraron 1681 individuos, 38 familias y 131 especies; las familias más representativas fueron Fabaceae, Euphorbiaceae y Moraceae. La diversidad fue similar en todas las zonas y la similitud florística osciló entre $31.3 \%$ y $53.3 \%$.

La vegetación ribereña y el suelo mostraron correlación positiva entre las variables altura de los árboles, $\mathrm{MO}$, $\mathrm{Nt}$ y $\mathrm{P}$, con tendencia a mejorar hacia la zona alta, siguiendo el gradiente de mayor a menor perturbación por las actividades humanas.

\section{RECONOCIMIENTOS}

Al proyecto financiado por FOMIX, Tabasco: "Evaluación de los indicadores de impacto ambiental identificados en el Estado de Tabasco", con Clave TAB-2009-C17-120755. Igualmente se corresponde al apoyo recibido por CONACYT en forma de beca otorgado al primer autor de este artículo bajo el número 417435 .

\section{REFERENCIAS}

Acosta, C., Mondragón, A. y Alvarado, H. (2008). Contribución de la flora arbórea de un sector del bosque ribereño “Los Letreros”, estado Trujillo, Venezuela. Revista Forestal Venezolana, 52(1), 21-31.

Aguirre, Z., Cabrera, O., Sánchez, A., Merino, B. y Maza B. (2003). Composición florística, endemismo y etnobotánica de la vegetación del sector oriental, parte baja del Parque Nacional Podocarpus. Lyonia, 3(1), 5-14.

Alvares, S. J. (2001). Descomposición y ciclo de nutrientes en ecosistemas terrestres de México. Acta Zoológica Mexicana, 1, 11-27.

Álvarez A., G., Ibáñez H., A., García C., N. E. y Almendros G., M. (2012). Almacenes de carbono y estabilidad de la materia orgánica del suelo en un agroecosistema cafetalero en la Sierra Sur de Oaxaca, México. Tropical and Subtropical Agroecoystems, 15, 611-620.

Aponte, C. (2011). Interacciones planta-suelo en un bosque mediterráneo. Ecosistemas, 20(2-3), 95-100.

Araujo, C. E., Closset, K. D., Gallet, M. E., Lenoir, J., Rêve, M., Hermy, M. y Decocq, G. (2013). Streams are efficient corridors for plants species in forest metacommunities. Journal of Applied Ecology, 50, 1152-1160. doi: $10.1111 / 1365-2664.12132$

Barba-Macías, E., Rangel-Mendoza, J. y Ramos-Reyes, R. (2006). Clasificación de los humedales de Tabasco mediante sistemas de información geográfica. Universidad y Ciencia, 22(2), 101-110.

Camacho, R. F., Trejo, I. y Bonfil, C. (2006). Estructura y composición de la vegetación ribereña de la Barranca del río Tembembe, Morelos, México. Boletín de la Sociedad Botánica de Mexico, 78, 17-31. 
Castañeda, M. B., Cabrera, A. F., Navarro, Y. y Vries, W. (2010). Procesamiento de datos y análisis estadísticos utilizando SPSS: Un libro práctico para investigadores $y$ administradores educativos. Porto Alegre: EDIPUCRS.

Ceccon, A. (2003). Los bosques ribereños y la restauración y conservación de las cuencas hidrográficas. Ciencias, 72, 46-53.

Chun, C. T., Zueng, S. C. y Chang, F. H. (2004). Relationships between soil properties and slope position in a lowland rain forest of southern Taiwan. Geoderma, 123, 131-142. doi:10.1016/j.geoderma.2004.01.031

Cortés-Castelán, J. C. e Islebe, G. A. (2005). Influencia de factores ambientales en la distribución de especies arbóreas en las selvas del sureste de México. Revista de Biología Tropical, 53(1-2), 115-133.

Decocq, G. (2002). Patterns of plant species and community diversity at different organization levels in a forested riparian landscape. Journal of Vegetation Science, 13, 91-106.

Diario Oficial de la Federación. (2002). NOM-021-RECNAT-2000. Que establece las especificaciones de fertilidad, salinidad y clasificación de suelos, estudio, muestreo y análisis. Distrito Federal, México: Semarnat. Recuperado de http://www.ordenjuridico.gob.mx/Documentos/ Federal/wo69255.pdf

Díaz, P. W. A., Rueda, J., Acosta, O., Martínez, O. y Castellanos, H. (2010). Composición florística del bosque ribereño del río San José, Reserva Forestal de Imataca, Estado Bolívar, Venezuela. Acta Botánica Benezolana, 33(1), $1-21$.

Ede, F. y Hunt, T. (2009). Understanding why weeds flourish in riparian zones. Land and Water Australia, 8, 14-15.

Fernández-Aláez, C., Fernández-Aláez, M. y García-Criado, F. (2005). Spatial distribution pattern of the riparian vegetation in a basin in the NW Spain. Plant Ecology, 179, 31-42.

Flores B., J. G., Batte B., C. y Dapara, J. (2002). Caracterización de la vegetación del río Undumo y su importancia para la conservación de la fauna silvestre. Ecología en Bolivia, 37(1), 23-48.

Gallardo, A., Covelo, F., Morillas, L. y Delgado, M. (2009). Ciclos de nutrientes y procesos edáficos en los ecosistemas terrestres: especificidades del caso mediterráneo y sus implicaciones para las relaciones suelo-planta. Ecosistemas, 18(2), 4-19.

García, L. y Vollmann, J. (2012). Caracterización de suelos a lo largo de un gradiente altitudinal en Ecuador. Revista Brasileña Ciéncias Agrárias, 7(3), 456-464.

García, E. (2004). Modificaciones al sistema de clasificación climática de Kôppen. Instituto de Geografía, UNAM. Recuperado de http://www.igeograf.unam.mx/sigg/utilidades/docs/pdfs/publicaciones/geo_siglo21/serie_lib/ modific_al_sis.pdf

Granados-Sánchez, D., Hernández-García, M. Á. y López-Ríos, G. F. (2006). Ecología de las zonas ribereñas. Revista Chapingo Serie Ciencias Forestales y del Ambiente, 12(1), 55-69.

Guevara, G., Reinoso, G., García, J. E., Franco, L. M., García, L. J., Yara, D. C., Briñez, N., Ocampo, M. L., Quintana, M. I., Pava, D. Y., Flórez, N. Y., Ávila, M. F., Hernández, E. E., Lozano, L. A., Guapucal, M., Borrero, D. A. y Olaya, E. J. (2008). Aportes para el análisis de ecosistemas fluviales: una visión desde ambientes ribereños. Tumbaga, 3, 109-127.

Hammer, Ø. (2012). Paleontological statistics. Reference manual. University of Olso. Recuperado de http://www. nhm2.uio.no/norlex/past/past_part1.pdf

Instituto Nacional de Estadística, Geografía e Historia. (2000). Cuaderno estadístico municipal de Tacotalpa, Tabasco. Recuperado de http://internet.contenidos.inegi.org.mx/ contenidos/productos/prod_serv/contenidos/espanol/bvinegi/productos/historicos/2104/702825931667/702 825931667_1.pdf

Johnson, W. C. (2002). Riparian vegetation diversity along regulated rivers: contribution of novel and relict habitats. Freshwater Biology, 47, 749-759.

Kaneko, Y. y Takada, T. (2008). Population dynamics and key stages in two Japanese riparian elements. En H. Sakio y T. Tamura (Eds.), Ecology of Riparian Forests in Japan: Disturbance, Life History and regeneration (pp. 91-105). Tokyo Berlin Heidelberg: Springer.

Kamrani, A., Jalili, A., Naqinezhad, A., Attar, F., Asgar, M. A. y Shaw, S. C. (2011). Relantionships between environ- 
mental variables and vegetation across mountain wetland sites, N. Iran. Biologia, 66(1), 76-87. doi: 10.2478/ s11756-010-0127-2

Llaven, M. V. (2013). Mamíferos de un bosque ribereño en la cuenca media del río Grijalva, Chiapas, México. Acta zoológica Mexicana, 29(2), 287-303.

López-Pérez, D., Castillo-Acosta, O., Zavala-Cruz, J y Hernández-Trejo, H. (2014). Estructura y composición florística de la vegetación secundaria en tres regiones de la sierra norte de Chiapas, México. Polibotánica, 37, 1-23.

Magurran, A. E. y McGill, B. J. (2011). Biological diversity: frontiers in measurement and assessment. Oxfor University Press.

Maldonado-Sánchez, E. A. y Maldonado-Mares. (2010). Estructura y diversidad arbórea de una selva alta perennifolia en Tacotalpa, Tabasco, México. Universidad y Ciencia, 26(3), 235-245.

Martínez-Ramos, M. y García-Orth, X. (2007). Sucesión ecológica y restauración de las selvas humedas. Boletín de la Sociedad Botánica de México, 80, 69-84.

Mingxi, J., Hongbing, D., Oinghua, C y Gang, W. (2005). Species richness in a riparian plant community along the banks on the Xiangxi River, The Three Georges region. Journal of Sustainable Development and Wordl Ecology, 12(1), 60-67.

Moffatt, S. F., McLachlan, S. M. y Kenkel, N. C. (2004). Impacts of land use on riparian forest along an urbanrural gradient in southern Manitoba. Plant Ecology, 174, 119-135.

Moreno, C. E. (2001). Métodos para medir la biodiversidad. Zaragoza Vol I. M \& T-Manuales y Tesis SEA.

Moreno, C. E. y Halffter, G. (2001). On the measure of sampling effort used in species accumulation curves. Journal of Applied Ecology, 38, 487-490.

Murray N., R. M., Bojórquez S., J. I., Hernández J., A., Orozco B., M. G., García P., J. D., Gómez A., R., Ontiveros G., H. M. y Aguirre O., J. (2011). Efecto de la materia orgánica sobre las propiedades físicas del suelo en un sistema agroforestal de la llanura costera norte de Nayarit, México. Revista Biociencias, 1(3), 27-35.

Ochoa-Gaona, S., Hernández-Vázquez, F. H., De Jong, B. H. J. y Gurri-García, F. D. (2007). Pérdida de diversidad florís- tica ante un gradiente de intensificación del sistema agrícola de roza-tumba-quema: un estudio de caso en la Selva Lacandona, Chiapas, México. Boletín de la Sociedad Botánica de México, 81, 65-80.

Ortiz-Pérez, M. A., Siebe, C. y Cram, S. (2005). Diferenciación ecogeográfica de Tabasco. En J. Bueno, F. Álvarez y S. Santiago (Eds.), Biodiversidad del Estado de Tabasco (pp. 305-322). México, D.F.: Instituto de Biología, UNAM-Conabio.

Palma-López, D. J., Cisneros D., J., Moreno C., E. y RincónRamírez, J. A. (2007). Suelos de Tabasco: Su uso y manejo sustentable. Villahermosa, Tabasco, México: Colegio de Postgraduados-Isprotab-Fuprotab.

Paul, E. A. (2007). Soil microbiology, ecology, and biochemistry. Amsterdam, Boston: Academic Press.

Pennington, T. D. y Sarukhán, J. (2005). Árboles Tropicales de México: Manual para la identificación de las principales especies. Fondo de Cultura Económica, México: UNAM.

Pérez, L. A., Sousa, S. M., Hanan, A. M., Chiang, F. y Tenorio, P. (2005). Vegetación Terrestre. En J. Bueno, F. Álvarez y S. Santiago (Eds.), Biodiversidad del Estado de Tabasco (pp. 65-110). México, D.F.: Instituto de Biología, UNAM-Conabio.

Porta, J., López, A. M. y Roquero, R. 2003. Edafología para la agricultura y el medio ambiente. Madrid: Mundi-Prensa.

Rodríguez, L. y Colonnelo, G. (2009). Caracterización florística de ambientes de la cuenca baja del río Cucurital, afluente del río Caroní, Estado Bolívar, Guayana Venezolana. Acta Amazónica, 39(1), 35-52.

Salgado-García., S., Palma-López, D. J., Castelán-Estrada, M., Lagunes-Espinoza, L. C. y Ortiz, L. H. (2013). Manual para el muestreo de suelos, plantas y aguas e interpretación de análisis para la producción sostenible de alimentos. México: Colegio de Posgraduados-ISPROTAB.

Sánchez, B., Ruíz, M. y Ruíz, M. M. (2005). Materia orgánica y actividad biológica del suelo en relación con la altitud, en la cuenca del río Maracay, estado Aragua. Agronomía Tropical, 55(4), 507-534.

Sarr, D. A. y Hibbs, D. E. (2007). Woody Riparian plant distributions in western Oregon, USA: comparing landscape and local scale factors. Plant Ecology, 190, 291-311. doi: 10.1007/s11258-006-9208-2 
Sepúlveda-Lozada, A., Geissen, V., Ochoa-Gaona, S., JarquínSánchez, A., Hernández-de-la-Cruz, S., Capetillo, E. y Zamora-Corelio, L. F. (2009). Influencia de tres tipos de vegetación ribereña en el control de la erosión fluvial en Pantanos de Centla, México. Revista de Biología Tropical, 57(4), 1153-1163.

Servicio Geológico Mexicano. (2005). Carta Geológico-Minera Villahermosa E15-8 Tabasco, Veracruz, Chiapas y Oaxaca. Recuperado de http://mapserver.sgm.gob.mx/ Cartas_Online/geologia/106_E15-8_GM.pdf

Sirombra, M. G. y Mesa, L. M. (2010). Composición florística y distribución de los bosques ribereños subtropicales andinos del río Lules, Tucumán, Argentina. Revista de Biología Tropical, 58(1), 449-510.

Smith, T. M. y Smith, R. L. (2007). Ecología. Madrid: Peason Educación.

Sunil, C., Somashekar, R. K. y Nagaraja, B. C. (2010). Riparian vegetation assessment of Cauvery River basin of South India. Environ Monit Assess, 170, 545-553. doi: 10.1007/ s10661-009-1256-3

Treviño G., E. J., Cavazos C., C. y Aguirre C., O. A. (2001). Distribución y estructura de los bosques de galería en dos ríos del Centro Sur de Nuevo León. Madera y Bosques, 7(1), 13-25. doi:10.21829/myb.2001.711315

Vázquez-Negrín, I., Castillo-Acosta, O., Valdez-Hernández, J.I., Zavala-Cruz J. y Martínez-Sánchez, J.L. (2011). Estructura y composición florística de la selva alta perennifolia en el Ejido Niños Héroes Tenosique, Tabasco, México. Polibotánica, 32, 41-61.

Wendt, T. y Lott, E. (1985). A new simple-leaved species of Recchia (Simaroubacea) from southeastern Mexico. Brittonia, 37(2), 219-225.
Yang, J., Dilts, T. E., Condon, L. A., Turner, P. L. y Weisberg, P. J. (2011). Longitudinal and transverse scale environmental influences on riparian vegetation across multiple levels of ecological organization. Landscape Ecology, 26, 381395. doi: 10.1007/s10980-010-9565-z

Yimer, F., Ledin, S. y Abdelkadir, A. (2006). Poperty variations in relation to topographic aspect and vegetation community in the south-eastern highlands of Ethiopia. Forest Ecology and Management, 232, 90-99.

Zarco-Espinosa, V. M., Valdez-Hernández, J. I., Ángeles-Pérez, G. y Castillo-Acosta, O (2010). Estructura y composición de la vegetación arbórea del Parque estatal Agua Blanca, Macuspana, Tabasco. Universidad y Ciencia, 26(1), 1-17. Zavala-Cruz, J., Palma-López, D. J., Fernández C., C. R., López C., A. y Shirma, T. E. (2011). Degradación y Conservación de Suelos en la Cuenca del río Grijalva, Tabasco. Colegio de Postgraduados, Secretaría de recursos Naturales y Protección Ambiental y PEMEX. Villahermosa, Tabasco. Recuperado de http://www.colpos.mx/ tabasco/2014/DEGRADACION\%20Y\%20CONSERVACION\%20DE\%20SUELOS.pdf

Zavala-Cruz, J., Jiménez-Ramírez, R., Palma-López, D. J., Bautista-Zúñiga, F., Gavi-Reyes, F. (2016). Paisajes geomorfológicos: base para el levantamiento de suelos en Tabasco, México: Ecosistemas y Recursos Agropecuarios, 3(8), 161-171.

Manuscrito recibido el 2 de junio de 2016.

Aceptado el 17 de enero de 2017.

Este documento se debe citar como:

Moreno-Jiménez, V., Castillo-Acosta, O., Gama-Campillo, L., ZavalaCruz, J. y Ortiz-Pérez, M. O. (2017). Relación de vegetación ribereña y propiedades del suelo en un afluente del río Tacotalpa, Tabasco, México.Maderay Bosques,23(1), 91-109. doi:10.21829/myb.2017.231510 
AnExo 1. Listado florístico de la vegetación ribereña registradas en un afluente del río Tacotalpa, Tabasco.

\begin{tabular}{|c|c|c|c|c|c|}
\hline \multirow[t]{2}{*}{ Familia } & \multirow[t]{2}{*}{ Especies } & \multirow[t]{2}{*}{ Nombre común } & \multicolumn{3}{|c|}{ Número de individuos } \\
\hline & & & B & $\mathrm{M}$ & A \\
\hline Anacardiaceae & Spondias mombin L. & Jobo & 4 & 8 & 14 \\
\hline Annonaceae & Annona squamosa $\mathrm{L}$. & Anonilla & - & 5 & - \\
\hline Apocynaceae & Stemmadenia donnell smithii (Rose) W. & Lecherillo & 1 & 5 & 4 \\
\hline Apocynaceae & Thevetia ahouai (L.) A. DC. & Bola de venado & 1 & - & - \\
\hline Araliaceae & Dendropanax arboreus (L.) March. & Desconocido & - & - & 3 \\
\hline Araliaceae & Dendropanax sp. & Zopo Blanco & - & 7 & 5 \\
\hline Arecacea & Chamaedorae elegans Mart. & Guaya & - & 2 & 25 \\
\hline Arecacea & Desmoncus chinantlensis Mart. & Ballíl & - & - & 1 \\
\hline Asteraceae & Tithonia diversifolia (Hemsl.) Gray. & Tanchiche & 5 & - & 5 \\
\hline Asteraceae & Vernonia patens Kunth & Malacate 3 & 3 & - & - \\
\hline Asteraceae & Vernonanthura patens (Kunt) H. Rob. & Malacate blanca & 10 & 44 & 1 \\
\hline Bignoniaceae & Amphitecna macrophyla (Seem.) & Jícara de montaña & - & 2 & 14 \\
\hline Bixaceae & Bixa orellana $L$. & Achiote & - & 1 & - \\
\hline Bombacaceae & Pachira aquatica Aubl. & Zapote de agua & - & - & 2 \\
\hline Bombacaceae & Qauraribea funebris (La Llave) Vischer & Molinillo & - & 2 & 7 \\
\hline Boraginaceae & Cordia alliodora (Ruiz \& Pav) Oken & Bojón & 2 & 2 & - \\
\hline Burceraceae & Bursera simaruba (L.) Sarg. & Palo mulato & 9 & 3 & 7 \\
\hline Caricaceae & Carica mexicana (A.DC) L. & Papaya silvestre & 5 & 2 & 4 \\
\hline Caesalpinioideae & Schizolobium parahybum (Vell.) S.F. Blake & Plumillo & - & 16 & 3 \\
\hline Cecropiaceae & Cecropia obtusifolia Bertol & Guarumo & 2 & 7 & 4 \\
\hline Euphorbiaceae & Acalypha sp.1 & Chamarrol & - & 31 & - \\
\hline Euphorbiaceae & Acalypha sp.2 & Chamarro2 & - & - & 64 \\
\hline Euphorbiaceae & Acalypha sp.3 & Chilpate & - & 3 & 10 \\
\hline Euphorbiaceae & Croton sp. & Patastillo & - & 2 & - \\
\hline Euphorbiaceae & Cnidoscolus multilobus (Pax) I.M.J. & Chaya pica & - & - & 6 \\
\hline Euphorbiaceae & Sapium sp. & Desconocido & - & - & 1 \\
\hline Euphorbiaceae & Euphorbiaceae (NI) & Desconocido & - & - & 4 \\
\hline Euphorbiaceae & Euphorbiaceae (NI) & Pata de mula & - & 1 & 1 \\
\hline Euphorbiaceae & Euphorbiaceae (NI) & Tabaco cimarrón & - & - & 4 \\
\hline Euphorbiaceae & Euphorbiaceae (NI) & Botoncillo negro & - & - & 1 \\
\hline Euphorbiaceae & Euphorbiaceae (NI) & Cola de ardilla & - & 2 & - \\
\hline Euphorbiaceae & Euphorbiaceae (NI) & Desconocido & - & - & 3 \\
\hline Flacourtiaceae & Hasseltia mexicana (A. Gray) Standl. & Capulincillo & 51 & 30 & - \\
\hline Flacourtiaceae & Casearia nítida Jacq. & Achiotillo & - & - & 4 \\
\hline Flacourtiaceae & Casearia sp.1 & Botoncillo & - & - & 9 \\
\hline Flacourtiaceae & Casearia sp.2 & Desconocido & - & - & 2 \\
\hline Flacourtiaceae & Flacourtiaceae (NI) & Carbonerol & 32 & 4 & 7 \\
\hline Flacourtiaceae & Flacourtiaceae (NI) & Desconocido & 3 & 9 & - \\
\hline Flacourtiaceae & Flacourtiaceae (NI) & Desconocido & - & - & 1 \\
\hline Flacourtiaceae & Flacourtiaceae (NI) & Desconocido & - & - & 1 \\
\hline Lauraceae & Lauraceae (NI) & Laurel hoja ancha & - & - & 5 \\
\hline Lauraceae & Nectandra sp & Laurelillo & - & 3 & - \\
\hline Lauraceae & Ocotea sp. & Laurel blanco & - & - & 5 \\
\hline
\end{tabular}


Anexo 1. Listado florístico de la vegetación ribereña registradas en un afluente del río Tacotalpa, Tabasco. Continuación...

\begin{tabular}{|c|c|c|c|c|c|}
\hline \multirow[t]{2}{*}{ Familia } & \multirow[t]{2}{*}{ Especies } & \multirow[t]{2}{*}{ Nombre común } & \multicolumn{3}{|c|}{ Número de individuos } \\
\hline & & & B & M & A \\
\hline Fabaceae & Acacia angustissima (Mill.) Kuntze & Charamusco2 & 39 & - & - \\
\hline Fabaceae & Acacia cornígera (L.) Willd. & Cornesuelo común & 24 & 7 & - \\
\hline Fabaceae & Acacia hayezii Benth. & Cola de lagarto & - & 14 & 6 \\
\hline Fabaceae & Acacia janzenii Ebinger et Seigler & Cornesuelo de montaña & 1 & 2 & 4 \\
\hline Fabaceae & Acacia sp. & Cabello de ángel & - & 1 & 2 \\
\hline Fabaceae & Erythrina americana Miller & Madre chontal & - & 4 & 9 \\
\hline Fabaceae & Gliricidia sepium (Jacq.) Steudel. & Cocohíte & 1 & 1 & - \\
\hline Fabaceae & Inga oerstediana Benth. & Chelele2 & - & 5 & - \\
\hline Fabaceae & Inga vera Willd. & Chelele & 18 & - & - \\
\hline Fabaceae & Inga sp. & Desconocido & - & - & 2 \\
\hline Fabaceae & Leucaena leucocephala (Lamarck) DeWitt. & Charamuscol & 6 & - & - \\
\hline Fabaceae & Lonchocarpus hondurensis Benth & Gusano & 2 & 28 & 9 \\
\hline Fabaceae & Lonchocarpus sp. & Desconocido & - & - & 1 \\
\hline Fabaceae & Pithecellobium micrandium Donn. Sm. & Desconocido & - & - & 1 \\
\hline Fabaceae & Pterocarpus rohrii Vahl. & Palo sangre & - & - & 1 \\
\hline Fabaceae & Vatairea lundellii (Standl.) Killip ex Record & Tinco & - & 1 & 5 \\
\hline Fabaceae & Fabaceae (NI) & Quelite & 61 & 33 & - \\
\hline Fabaceae & Zigia sp.1 & Pinolillo blanco & - & - & 1 \\
\hline Fabaceae & Zigia sp.2 & Pinolillo & - & 1 & - \\
\hline Fabaceae & Fabaceae (NI) & Desconocido & - & - & 3 \\
\hline Fabaceae & Fabaceae (NI) & Desconocido & - & - & 3 \\
\hline Malvaceae & Hampea nutricia Fryxell & Majagua amarilla & - & 1 & 1 \\
\hline Malvaceae & Hibiscus sp. & Tulipancillo & 2 & - & - \\
\hline Malvaceae & Robinsonella mirandae Gómez Pompa & Majagua blanca & 9 & 5 & 3 \\
\hline Meliaceae & Cedrela odorata L. & Cedro & 5 & - & 4 \\
\hline Meliaceae & Guarea grandiflora C. DC. & Chelele de montaña & 1 & 3 & 4 \\
\hline Meliaceae & Guarea sp. & Cacaté de montaña & - & - & 5 \\
\hline Meliaceae & Trichilia havanensis Jacq. & Coshigue & 29 & 7 & - \\
\hline Melastomataceae & Miconia argéntea (Sw.) DC & Hoja de lata & - & 1 & - \\
\hline Monimiaceae & Monimiaceae (NI) & Desconocido & - & - & 1 \\
\hline Moraceae & Castilla elástica Cerv. & Hule & - & 1 & 1 \\
\hline Moraceae & Ficus insipida Willd. & Amatillo & 10 & 10 & 2 \\
\hline Moraceae & Ficus yoponensis Desv. & Amate & 6 & - & - \\
\hline Moraceae & Ficus sp.1 & Matapalo & 1 & - & 1 \\
\hline Moraceae & Ficus sp.2 & Desconocido & - & - & 5 \\
\hline Moraceae & Brosimum guianense (Aubl.) Huber & Hosh rojo & - & 1 & 10 \\
\hline Moraceae & Brosimum costaricanum Liebm. & Hosh blanco & - & 2 & 20 \\
\hline Moraceae & Poulsenia armata (Miq.) Standl. & Carne de pescado & - & 7 & 1 \\
\hline Moraceae & Pseudolmedia sp. & Baril & - & - & 1 \\
\hline Moraceae & Trophis racemosa (L.) & Ramoncillo & 1 & 2 & - \\
\hline Myrtaceae & Eugenia acapulcensis Steud. & Guayabillo2 & - & 1 & - \\
\hline Myrtaceae & Eugenia sp.1 & Guayabillo3 & - & 1 & - \\
\hline Myrtaceae & Eugenia sp.2 & Tomatillo del río & 2 & - & - \\
\hline
\end{tabular}


AnExo 1. Listado florístico de la vegetación ribereña registradas en un afluente del río Tacotalpa, Tabasco. Continuación...

\begin{tabular}{|c|c|c|c|c|c|}
\hline \multirow[t]{2}{*}{ Familia } & \multirow[t]{2}{*}{ Especies } & \multirow[t]{2}{*}{ Nombre común } & \multicolumn{3}{|c|}{ Número de individuos } \\
\hline & & & $\mathrm{B}$ & $M$ & $A$ \\
\hline Myrtaceae & Myrcia splendens (Sw.) & Sauce & 1 & - & - \\
\hline Myrtaceae & Psidium guajava L. & Guayaba criollo & 5 & - & - \\
\hline Myrtaceae & Myrtaceae (NI) & Sierrilla & - & - & 1 \\
\hline Myrsinaceae & Ardisia paschalis Donn. Sm. & Coshigue de montaña & - & 2 & - \\
\hline Piperaceae & Piper amalago L. & Malacate tallo rugoso & 5 & 25 & 4 \\
\hline Piperaceae & Piper arboreum Aubl. & Malacate tallo verde-lisa & 36 & 46 & 13 \\
\hline Piperaceae & Piper auritum Kunth & Momo & 21 & 84 & 7 \\
\hline Piperaceae & Piper umbellatum L. & Momo Cimarrón & 1 & - & - \\
\hline Piperaceae & Piper sp.1 & Malacate tallo manchado & 3 & 24 & 4 \\
\hline Piperaceae & Pipersp.2 & Tokoy & 1 & 1 & - \\
\hline Piperaceae & Piperaceae (NI) & Barba de tuza & - & - & 5 \\
\hline Rhamnaceae & Colubrina arborescens (Mill.) Sarg. & Desconocido & - & 1 & - \\
\hline Rubiaceae & Blepharidium mexicanum Standl. & Popiste & - & 12 & - \\
\hline Rubiaceae & Hamelia patens Jacq. & Coralillo & - & - & 15 \\
\hline Rubiaceae & Rubiaceae (NI) & Desconocido & - & - & 2 \\
\hline Rubiaceae & Simira salvadorensis (Standl.) Steyerm. & Popiste de montaña & - & 1 & - \\
\hline Rutaceae & Citrus aurantium L. & Naranja & 6 & - & - \\
\hline Rutaceae & Citrus aurantifolia (Christem.) & Limón criollo & 2 & 5 & - \\
\hline Rutaceae & Citrus sp.1 & Limón real & 2 & 1 & - \\
\hline Rutaceae & Citrus reticulata & Mandarina & 1 & - & - \\
\hline Rutaceae & Esenbeckia sp.1 & Bálsamo o Limoncillo & - & 10 & 12 \\
\hline Rutaceae & Esenbeckia sp.2 & Ballester & - & - & 2 \\
\hline Rutaceae & Zanthoxiylum kellermanii P. Wilson & Abrojo & 2 & - & - \\
\hline Rutaceae & Zanthoxylum sp. & Palo duro & - & - & 1 \\
\hline Sapindaceae & Cupania dentata Moc. \& Sessé ex DC. & Quiebra hacha & 19 & 2 & 1 \\
\hline Sapindaceae & Matayba oppositifolia A. Rich Britton & Carbonero2 & - & 4 & 2 \\
\hline Simaroubaceae & Recchia simplicifolia T. Wendt. & Tomatillo & - & - & 48 \\
\hline Solanaceae & Cestrum nocturnum L. & Amargoso & 1 & 8 & 5 \\
\hline Solanaceae & Solanum torvum Sw. & Candelero & 1 & - & - \\
\hline Solanaceae & Solanum sp.1 & Chilillol & 1 & 5 & - \\
\hline Solanaceae & Solanum sp.2 & Chilillo2 & - & 1 & - \\
\hline Solanaceae & Solanum sp.3 & Desconocido & 1 & - & - \\
\hline Solanaceae & Witheringia solanácea L’Hér. & Cuñai & - & 6 & 2 \\
\hline Solanaceae & Solanaceae (NI) & Guayabillol & 6 & - & 1 \\
\hline Solanaceae & Solanaceae (NI) & Escobillo2 & 3 & - & - \\
\hline Sterculiaceae & Guazuma ulmifolia Lam. & Guácimo & 7 & 12 & - \\
\hline Sterculiaceae & Sterculia sp. & Bellota & - & - & 5 \\
\hline Tiliaceae & Belotia sp. & Azota caballo & 5 & 57 & - \\
\hline Tiliaceae & Mortoniodendron anisophyllum (Standl.) & Zopo Amarillo & - & 4 & 5 \\
\hline Ulmaceae & Trema micrantha (L.) Blume & Capulín & 5 & - & - \\
\hline Urticaceae & Urera caracasana (Jacq.) Griseb. & Cholagogue & - & 16 & 22 \\
\hline Urticaceae & Urera elata (Sw.) Griseb. & Palo de agua & - & 16 & 8 \\
\hline Verbenacea & Cornutia pyramidata L. & Pangajei & 2 & 5 & 12 \\
\hline
\end{tabular}


Anexo 1. Listado florístico de la vegetación ribereña registradas en un afluente del río Tacotalpa, Tabasco. Continuación...

\begin{tabular}{|c|c|c|c|c|c|}
\hline \multirow[t]{2}{*}{ Familia } & \multirow[t]{2}{*}{ Especies } & \multirow[t]{2}{*}{ Nombre común } & \multicolumn{3}{|c|}{ Número de individuos } \\
\hline & & & B & $M$ & $A$ \\
\hline Verbenacea & Verbenacea (NI) & Escobillo 1 & 1 & 7 & - \\
\hline \multirow[t]{2}{*}{ Zapotaceae } & Manilkara sp. & Chicozapote de montaña & - & - & 2 \\
\hline & Total individuos(N) & & 484 & 685 & 512 \\
\hline
\end{tabular}

$\mathrm{NI}=$ No identificado. Sombreados= Especies que estuvieron distribuidas en las tres zonas: baja (B), media (M) y alta (A). spl, sp2...especies diferentes del mismo género. 